\title{
汉语存现句及其阿语翻译
}

الجملة الوجودية في اللغة الصينية وترجمتها إلى اللغة العربية

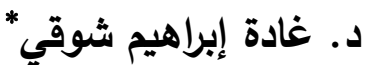

ghada.amr2010@gmail.com

ملخص

تعد اللغة العربية واللغة الصينية من أعرق اللغات في العالم وأكثرها تعقيدا. وكما يوجد بعض التشابهات بين اللغتين ،كذلك يوجد بعض الاختلافات بينهما. وتعد الجملة الوجودية أحد الأنماط اللغوية الثائع استخدامها في اللغة الصينية، وهذا النوع الخاص من الجمل لا يوجد مثيله في اللغة العربية، بمعنى أنه لا يوجد مسمى "جملة الوجود أو "الجملة الوجودية" في اللغة العربية، بل يتم التعبير عنه بأساليب لغوية معينة، مثل الفعل "يوجد"، وظرف الزمان وظرف المكان وتركيب الجار والمجرور وغيرها من التراكيب اللغوية. لذلى

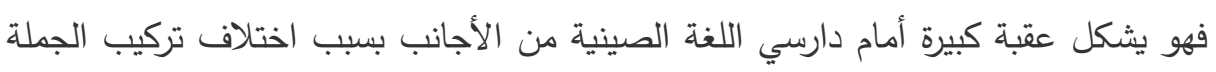
ما بين اللغة العربية واللغة الصينية. ومن خلال هذا البحث، سيقوم الباحث بدراسة تعريف

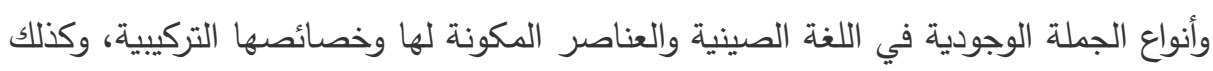
إجراء تحليل دقيق لطريقة ترجمتها إلى اللغة العربية. وذلك بهدف جعل الطالب قادرا على فهم طبيعة الجملة الوجودية وتركيبتها وطريقة ترجمتها إلى اللغة العربية، وكذلك إدرالك التثابهات والاختلافات بين اللغتين. كلمات مفتاحية: الجملة الوجودية - اللغة الصينية - اللغة العربية * مدرس بقسم اللغة الصينيةـ كلية الآداب - جامعة القاهرة (الجملة الوجودية في اللغة الصينية وترجمتها إلى اللغة العربية...) د. غادة إبر اهيم شوفي 
摘要: 汉语存现句是汉语中相当常见的一类句式。这种 特别的句式在结构上跟阿拉伯语的表达方式不一致, 因 此, 对非中国本土学生来说, 存现句是一种无法避免的 难度。通过这篇论文, 笔者将探索研究汉语存现句的定 义、分类与结构特点, 对其阿译进行详细的分析, 以便 能让学生理解存现句的结构及其阿语翻译。

关键词: 存现句; 汉语; 阿语; 存在句; 隐现句; 表达 方式; 阿译

\section{0 汉语存现句的定义、组成部分及分类}

\section{1 存现句的定义}

存现句是汉语的一种特别的句式, 从意义得名。倘 若要表达 “存在” 或 “消失” 的意义, 就应该使用这一 项结构特殊的存现句。

中国语法学家的以往研究未呈太大的差异。其中黄 伯荣、廖序东的《现代汉语》明确界定了存现句: “存现 句表示什么地方存在、出现或消失了什么人或物, 是用 来描写环境或景物的一种特定句式。” 1 同样, 王初艳在

${ }^{1}$ 《现代汉语 $[M]$ 》, 黄伯荣, 廖序东. 北京: 高等教育出版社, 2007。

(الجملة الوجودية في اللغة الصينية وترجمتها إلى اللغة العربية...) د. غادة إبر اهيم شوقي 
《英汉存现句对比研究》一书中指出: “存现句是表示事 物、动作或状态的存在、出现、消失的一类句子。”

齐沪扬教授也在其书《对外汉语教学语法》中如此 界定了存现句: “存现句是用来表示某人或某事物存在、 出现或消失于某处或某时的句子。它的句首为表示处所、 时间的词或词组, 说明某处、某时存在、出现或消失某 人某事物。” 3

值得一提的是晋文琦在其论文中给了存现句较为详 细的定义: “存在句是表示人或事物存在的句子。存在 句可以描写人物的穿着、打扮和客观环境, 表示人或事 物存在的一种状态。常用的句式结构是 “处所词语+动词 + 名词” , 例如: 桌子上放着几支笔、停车场停着几辆 车。” 4

就阿语来看, 阿语中并没有 “存现句” 这一语法概 念, 因此, 存现句在阿语中却没有具体定义, 只不过是 一种普通的句子而已。通过这篇论文, 我们将挖掘出汉 语存现句在阿语中的表达方式及二者之间的异同之处。

2 《英汉存现句对比研究》, 王初艳, 学科探索, 2019 年, 第 11 期。 3 《对外汉语教学语法》, 齐沪扬 主编, 复旦博学, 2005 年 9 月, 第一版。

《存现句分析及教学建议》, 晋文琦, 哈尔滨师范大学, 黑龙江。

(الجملة الوجودية في اللغة الصينية وترجمتها إلى اللغة العربية...) د. غادة إبراهيم شوقي 


\section{2 组成部分}

齐沪扬认为存现句的基本结构为四个成分:

“表示处所、时间的词或词组（状语）+表示存在、出 现或消失的动词（谓语）十动态助词+表示存在、出现或消 失的名词（人或事物）（宾语）。” 5 不过, 动态助词可 有可无, 只用表示存在、出现或消失的动词而已。因此王 初艳在《英汉存现句对比研究》 ${ }^{6}$ 以存现句的基本结构视为 三个组成部分, 即是: “方位名词短语 + 存现动词 + 存 现主体短语”。其可看成由三个成分构成, 即表处所、范 围或时间的短语、表存在、出现或消失的动词和存现句的 论述主体。我们可以以下面的图形对存现句的成分加以说 明。

\section{处所/} 时间

\section{方位名词短语}

存现/ 消失
动词
主体

名词

例如:
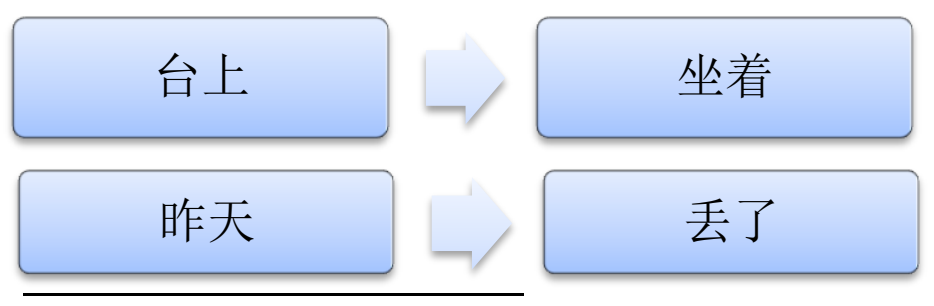

主席团

钱包

${ }^{5}$ 《对外汉语教学语法》, 齐沪扬 主编, 复旦博学, 2005 年 9 月, 第一版。

${ }^{6}$ 《英汉存现句对比研究》, 王初艳, 学科探索, 2019 年, 第 11 期。

(الجملة الوجودية في اللغة الصينية وترجمتها إلى اللغة العربية...) د. غادة إبراهيم شوقي 


\section{3 存现句的分类}

学界依据不同的理论基础, 给存现句出了不同的分 类结果, 刘月华认为 “存现句可以分为两类：一类是表 示人或事物存在的，叫存在句; 一类是表示人或事物的 出现、消失的，有人称隐现句。” 7

\section{1.存在句}

-即是表示人或事物存在的句子。

・例如：墙上挂着一幅画。

\section{2.隐现句}

・即是表示人或事物的出现、消失的句子。

・例如: 昨天丢了钱包。

\section{0 汉语存现句的阿译}

下面在讲解存现句的句式与结构特点的同时, 笔者 还将分析研究如何使用阿语来表达其意思。

\section{1 存在句的阿译}

\section{（一）表达功能与句式}

在汉语里, 当需要描写客观环境、人物的姿态和存在 的方式的时候, 就可以用存在句。这种句子是用于说明 某个处所存在着一个人或事物。其句式一般如下:

7 《实用现代汉语语法》, 刘月华等著, 商务印书馆, 北京, 2004 年。

(الجملة الوجودية في اللغة الصينية وترجمتها إلى اللغة العربية...) د. غادة إبر اهيم شوقي 


\section{主语 \\ （处所）}

动词

宾语

（人/事物）

而阿拉伯语中并没有 “存在句” 这一概念, 因此, 我 们将按结构去探索研究怎么用阿拉伯语来表达汉语存现 句, 看看汉语与阿语是否有同样的表达方式和结构。下面 分析一下汉语存在句的阿译:

(1)楼前 是 学校的图书馆。 主语 动词 宾语

(2)包里 有 很多钱。 主语 动词 宾语

(3)墙上挂着画儿。 主语 动词 宾语
توجد مكتبة الكلية أماح المبني. 偏地点副词施动 يوجد الكثير من المال في الحقيبة. 介词短语介词短语 施 动 توجد لوحة معلقة على الحائط. 介词短语定语施 动

(4)路上 走着 很多人。 يوجد أناس كثيرون سائرون في الثارع 主语 动词 宾语 介词短语定语 定语 施动

看上述的例子就能注意到，例(1)(2)中的汉语存在句 是由主语、动词和宾语组成的, 二者的动词是 “是” 和 “有”，都说明某个人或事物的存在。当将其翻成阿语 时, 就使用动词、施事者和介词短语来表达出来。

尽管例(3) (4)中的存在句也是由主语、动词和宾语组 成的，可是二者的阿语表达方式跟例(1)不太一致。因 为存在句中的动词说明某种动作或状态, 如 “挂” 和 
“走”，所以在阿语中的表达方式还加上了 “定语” 来 描写此动作或状态。

\section{（二）结构特点}

接下来, 我们就从汉语存在句的结构特点的角度来详细 地分析其阿语表达方式:

\section{1. 存在句的处所词}

存在句的句首往往是处所词, 作句子的主语, 它就是 被描写的对象。处所词一般由处所的名词、方位词、 “名词+方位词”、处所代词充任。

例如:

(1)教室里坐着十几个学生。（名词+方位词）

主语 يوجد عشرات الطلاب جالسون في قاعة الدرس. 介词短语

(2)墙上挂着一双春联。

(处所的名词) 主语 كلمات الربيع معلقة على الحائط. 介词短语

(3)上有天堂， 下有苏杭。（方位词） 主语主语 الجنة في الأعلى ، وخانغ جو بالأسفل. 介词短语 介词短语 
(4)抽庶里放着一本汉语书, 是谁的? (处所的代词) 主语

\section{يوجد كتاب لغة صينية موضوع في الارج، لمن هذا؟ 介词短语}

从上述看, 例子中的不同汉语处所词 “教室里”、 “家门口”、“上”、“抽屉里”都放在句首作主语。 当我们将其翻成阿语时, 就会使用介词和名词来表示处 所, 放在句尾。例如: 介词 “准在”、“上”、 “在” “里” 和名词 “洝”教室” 、

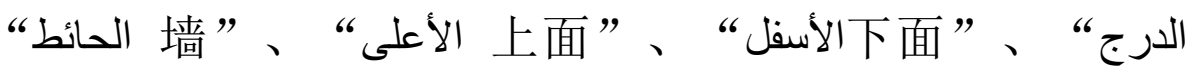
抽席”。

总之, 汉语存在句的处所词作句子的主语, 位于句首。 相反，阿语中的处所短语一般放在句尾。

\section{2. 存在句的动词}

存在句的动词位于处所词后面, 作谓语, 有以下四种 动词类型:

（1）表示人或事物的运动或姿势的动词，如: “走”、“站”、“坐”、“围”、“躺” 等 等。

（2）表示人对事物进行的动作的动词，如：“挂”、 “放”、“刻”、“贴”等等。

(الجملة الوجودية في اللغة الصينية وترجمتها إلى اللغة العربية...) د. غادة إبر اهيم شوقي 
在存在句中, 动词后一般有动态助词 “着” , 以便表 示人或事物的姿态或样子。如:

(1)家门口站着一个孩子。 يوجد طفل واقف لدى باب البيت. 动词 定语 动词

(2)路上走着很多人。 يوجد أناس كثيرون سائرون في الثنارع. 动词 定语 动词

(3)石壁上刻着很多图案。

动词

$$
\text { يوجد الكثير من الرسومات المحفورة على الحائط الحجري. }
$$

看到上述的例子, 就可以注意到汉语存在句的动词 “站”、“走” 和 “刻” 都在阿译中用定语 “国站 着”、“س走着”、“被刻着的ة 来描写对象 “侅子”、“图案”。同时, 要 使用动词 “客” 来表示存在的意思, 并放在句首。

（3）动词 “是”：可以用动词 “是” 表示存在, 和其动词比较, 带 “是” 的存在句还保留一点判断的 意味。例如:

(4)远处是一片绿地。 توجد مسطحات خضر اء بالمكان البعيد. 动词 动词 (الجملة الوجودية في اللغة الصينية وترجمتها إلى اللغة العربية...) د. غادة إبر اهيم شوقي 
(5)教室对面是学校图书馆。. توجع مكتبة الكلية أمام قاعة الدرس.

动词

动词

6国东边是上海。

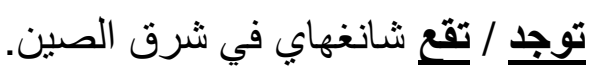

动词

动词

例(4)(5)都指某处的位置 “，这里，“是” 相当于阿 语中的动词 “ يو يقد في 存在” 或 “位于”, 都处于句 首, 而汉语存在句的动词位于主语后面作谓语。

（4）动词 “有” : 这就是最直接的、最基本的存在 句, 就意味着 “某处有什么”，不表示人或事 物的动作或姿态，仅仅表示 “存在” 的意思。 例如:

(7)前面有一座大山。 يوجد جبل كبير بالأمام.

动词 动词

8)房间里有很多椅子。 توجد الكثير من المقاعد في الغرفة. 动词 动词

例(7) (8)也是指某处的位置 “，“有” 相当于阿语 中的动词 “ يو يو 存在”, 都处于句首, 而汉语存在 句的动词位于主语后面。

(الجملة الوجودية في اللغة الصينية وترجمتها إلى اللغة العربية...) د. غادة إبر اهيم شوقي 


\section{3. 存在句的宾语}

存在句的宾语就是这种句子所要说明的新信息, 由 名词充任, 不过名词一般不能单用, 而前面往往有数量 词或定语, 往往表示不确定的人或事物。

例如:

(1)窗户上贴着一个“福”字。 توجد كلمة "سعادة" ملصقة على النافذة. 宾语

施

(2) 石上刻着一束花。

$$
\text { توجد زهرة مرسومة على الحجر. }
$$
宾语

对举时, 存在句的宾语前可以不用定语, 如:

(3)山上长着树。

宾语

(4) 埃及的东边是红海。 宾语

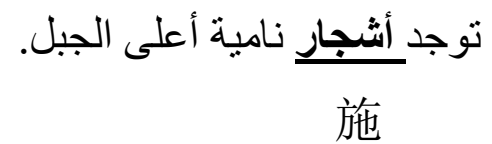

يقع البحر الأحمر في شرق مصر. 施

上述的例子指明汉语存在句中的宾语 “一个 “福” 字”、“一束花”、“树” 和 “红海” , 都放在句尾作 宾语, 不论是否确定的人或事物, 都以阿语的施事者来 表达出来, 并处于动词后面。

\section{2 隐现句的阿译}

隐现句是存现句的另一种，是表示某人或事物的出 现、消失的句子。这种句子表示出现的更常见。

$$
\text { (الجملة الوجودية في اللغة الصينية وترجمتها إلى اللغة العربية...) د. غادة إبر اهيم شوقي }
$$




\section{（一）表达功能与句式}

在汉语里, 当要叙述在某一处所或某一时间有人或 事物出现或消失的时候, 就可以使用隐现句来表示此意 思。一般用下列句式:

\section{处所词/时间词}
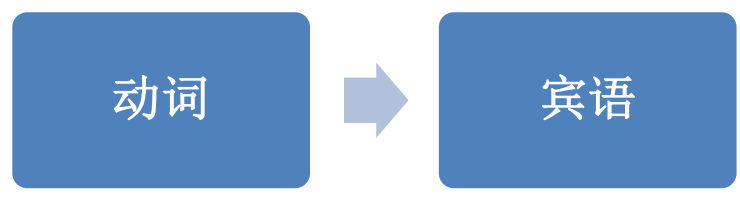

例如:
(1)家里 来了一位客人。
أتى ضيف بـ إلى بيتنا. 主语 动词 宾语
介词短语 施 动
(2)现在 发生了一件大事。

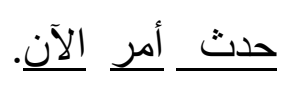
主语 动词 宾语
时间副词 施 动
(3)他们家 死了一个人。
主语 动词 宾语

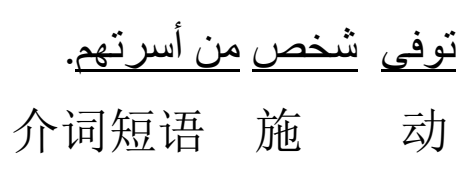

可见，与存在句相比，隐现句句首可以用或加上时 间词，而存在句一般不用时间词在句首。

同样，阿拉伯语中也没有 “隐现句”，而使用若干 的动词和语法结构以表达出来。分析上述的例子就可以 发现, 汉语存在句是由主语、动词和宾语组成的。当将 其翻成阿语时，就使用动词、施事者和介词短语或时间 副词来表达出来。

(الجملة الوجودية في اللغة الصينية وترجمتها إلى اللغة العربية...) د. غادة إبر اهيم شوقي 


\section{（二）结构特点}

接下来, 我们将按结构去探索研究怎么用阿拉伯语 来表达汉语隐现句, 并挖掘汉语隐现句的阿语表达方式。

\section{1. 句首的处所词/时间词}

隐现句的处所词或时间词也是跟存在句的处所词一 样, 都位于句首, 并表示出现或消失的处所或时间。例 如:

(1)迎面跑过来了一个孩子。. ركض طفل نحونا من الأماد. 主语 介词短语

(2) 天空飞过一群大雁。 طار سرب من الأوز في السماء. 主语 介词短语

(3)今天丢了钱包。 فقدت المحفظة اليوم. 主语 时间副词 فقدت اليوم المحفظة. 时间副词

(4) 昨天下雪了。 主语
تساقط الثليج البارحة.

时间副词

由上述的例子可以看出, 汉语隐现句的主语是处所 词时, 就翻成阿语中的介词短语

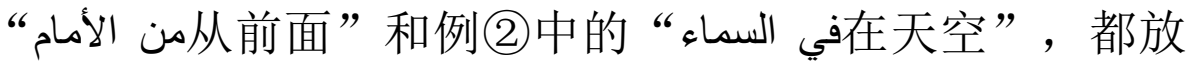
在句尾作介词短语, 与汉语隐现句相反, 处所词都放在 句首作主语。

(الجملة الوجودية في اللغة الصينية وترجمتها إلى اللغة العربية...) د. غادة إبر اهيم شوقي 
另外, 汉语隐现句主语由时间词充当时, 就相当于

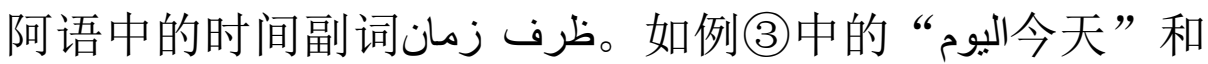
例(4)中的 “㫧昨天” , 都放在句尾, 有时阿语的时间 副词可以放在动词后面。

\section{2. 隐现句的动词}

（1）隐现句的动词多为不及物动词, 一般分为两 种: 一种是表示人或事物的出现、消失的, 如: “冒”、“浮现”、“死”、“飘”、“响”等, 另一种是表示人或事物的移动的，如: “走”、 “开”、“来”、“跑”等。

(2) 在隐现句子中, 动词后常带趋向补语、结果 补语和动态助词 “了”。例如:

(1)昨天我们班来了一个新同学。(表示移动)

$$
\text { أنى إلى صفنا طالب جديد البارحة. }
$$

(2)脸上浮现出喜悦的神情。（表示出现）

动

$$
\text { ظهرت على وجهه ملامح السرور. }
$$

(3)我朋友家死了一只猫。（表示消失） 
看上述的例子, 就可以注意到汉语隐现句的动词 “来”、“浮现出”、“死” 相当于阿语动词 “㳊来 了”、“ت “出现了”、“ت “死了”。可是汉语与阿 语的动词位置不一致; 汉语隐现句的动词位于主语后面 作谓语，而阿语中的动词位于句首。

\section{3. 隐现句的宾语}

即是隐现句中所出现或消失的对象，一般是不确 定的人或事物, 也是隐现句的新信息, 前面往往带数量 词或定语。例如:

(1)屋里走出了一位医生。 宾语

(2)天上飞过一只小岛。 宾语

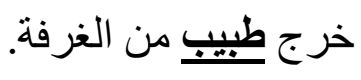

施事者

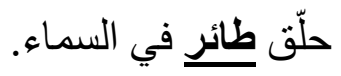

施事者

(3)新华饭店门口开来了一辆汽车。

\section{宾语 . أنت سيارة أمام مدخل فندق شين خو \\ 施事者}

有时, 隐现句的宾语不带数量词, 只用 “了” 在句 尾, 具有发生变化的语气。如:

(4)昨天下雨了。

宾语

(5)张家死人了。

宾语

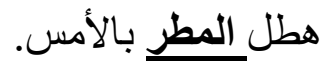

施事者

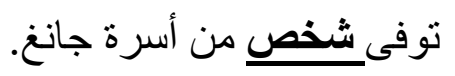

施事者

(الجملة الوجودية في اللغة الصينية وترجمتها إلى اللغة العربية...) د. غادة إبر اهيم شوقي 
(6)来客人了。

جاءنا ضيف.

宾语

施事者

显而可见，上述的隐现句中的宾语一般是动作的 发出者, 但是因其位于动词后面而即为宾语。果然, 当 我们把隐现句翻成阿语时, 即能发现隐现句的宾语在阿 语的句子却变成施事者。例(1)(2)(3)中的宾语 “一位医 生”、“一只小鸟”、“一辆汽车” 就相当于阿语中的

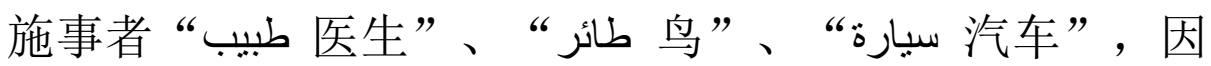
为动作是由它们进行的; “医生走出了”、“乌飞过”、 “汽车开来了”。同样，例(4)(5)6的宾语 “雨”、 “人”、“客人”在阿译中都变成施事者: “吕雨”、 “ش人”、“ف客人”, 因为动作由它们发生: “雨下了”、“人死了”、“客人来了”。

\section{0 汉语存现句的阿译研究小结}

（一）总体上讲, 汉语存现句是汉语中的一种特别的 语法结构，可是阿语中没有存现句这一概念， 而使用其它语言手段来表达同样的意义。如: 动词 “䒠存在”、介词短语、时间副词、地 点副词、定语等。

\section{（二）存在句:}

- 格式为: 主语+动词+宾语 


\section{- 阿译格式:}

- 动词+ 施事者 + 介词短语

- 动词+施事者 + 地点副词 + 偏次

- 动词+施事者+定语 + 介词短语

- 存在句与其阿译结构上的区别:

（1）存在句的处所词：往往位于句首，作主语。 但在阿译中，处所词位于句尾，作介词短 语。

（2）存在句的动词: 位于处所词后面, 而在阿 译中将其翻成定语，同时，加上其它动词 “ “و 存在”，位于句首。

由动词 “是” 充当的谓语, 即翻成 “ 存在” 或 “ يقع في 位于”; 由动词 “有”充 当的谓语, 即翻成 “足存在”。

（3）存在句的宾语: 位于句尾, 而在阿译中就 转成施事者，位于动词后面。

\section{（三）隐现句:}

- 格式为: 主语+动词+宾语

- 阿译格式:

- 动词 + 施事者 + 介词短语

- 动词 + 施事者+时间副词 


\section{- 隐现句与其阿译结构上的区别:}

（1）隐现句的处所词: 往往位于句首,

作主语。但在阿译中, 处所词位于

句尾，作介词短语。

（2）隐现句的时间词：往往位于句首, 作主语。但在阿译中, 时间词位于 句尾, 有时还可以放在动词后面, 作时间副词。

（3）隐现句的动词: 位于处所词后面, 作谓语, 而在阿译中的动词一般放在句 首。

（4）隐现句的宾语: 位于句尾, 而在阿 译中即成为施事者, 位于动词后面。

\section{参考书目}

\section{一、中文参考文献:}

\section{著作类}

1 -《现代汉语 $[\mathrm{M}] 》$, 黄伯荣, 廖序东. 北京：高等教 育出版社，2007。

2-《实用现代汉语语法》, 刘月华等著, 商务印书馆, 北京，2004 年。 


\section{0 ) 期刊类}

1- 《英汉存现句对比研究》, 王初艳, 学科探索, 2019

年，第 11 期。

2- 《对外汉语教学语法》, 齐沪扬 主编, 复旦博学, 2005 年 9 月, 第一版。

3-《存现句分析及教学建议》, 晋文琦, 哈尔滨师范大 学, 黑龙江, http://www. cnki. net。

\section{二、阿拉伯语参考文献:}

1- نحو اللغة العربية، دكتور محمد أسعد النادري، المكتبة العصرية، صبدا،

$$
\text { بيروت، } 2008 .
$$

2- ملخص قو اعد اللغة العربية، فؤاد نعمة، مطبعة نهضة مصر ، عام

.1973

(الجملة الوجودية في اللغة الصينية وترجمتها إلى اللغة العربية...) د. غادة إبراهيم شوقي 